\title{
Depressive Symptoms Among Adolescents: Testing Vulnerability-Stress and Protective Models in the Context of COVID-19
}

\author{
Tracy R. G. Gladstone ${ }^{1}$ (1) $\cdot$ Jennifer A. J. Schwartz ${ }^{2} \cdot$ Patrick Pössel $^{3} \cdot$ Amanda M. Richer $^{1} \cdot$ Katherine R. Buchholz $^{1}$. \\ L. Sophia Rintell ${ }^{1}$
}

Accepted: 27 June 2021 / Published online: 7 July 2021

(c) The Author(s), under exclusive licence to Springer Science+Business Media, LLC, part of Springer Nature 2021

\begin{abstract}
Adolescents who experience negative life events may be at risk for depression, particularly those with psychosocial vulnerabilities. We investigate longitudinally the impact of vulnerability/protective factors on the relation between a large-scale negative life event, the COVID-19 pandemic, and depressive symptoms. Adolescents ( $\mathrm{N}=228, \mathrm{M}_{\text {age }}=14.5$ years, $53 \%$ female, $73 \%$ white) self-reported depressive symptoms 2-4 months before the pandemic (Time 1), and again 2 months following stay-at-home orders (Time 2). At T2, adolescents also completed measures of vulnerability, protective factors, and COVID19-related distress. Depressive symptoms increased at T2, and COVID-19 distress interacted with resilience and negative cognitive style in predicting increases in $\mathrm{T} 2$ depression. Focusing on vulnerability and protective factors in adolescents distressed by large scale negative life events appears crucial.
\end{abstract}

Keywords Adolescent · Depression · COVID-19 $\cdot$ Resilience $\cdot$ Cognitive styles

\section{Introduction}

The developmental period of adolescence is characterized by significant social and biological changes that predispose youth to a range of behavioral health concerns [1]. When large-scale negative life events occur during this time, adolescents become vulnerable to the emergence of mental health concerns such as depression [2]. The COVID-19 pandemic, for example, is a negative life event for many adolescents as school closures, social distancing, and other safety procedures have altered their day-to-day lives [3]. The social isolation, online learning, and fear associated with the pandemic have the potential to significantly impact mental health [4]. As a result, clinicians and researchers have highlighted the potential mental health effects of COVID-19 on youth $[2,5]$. Cross-sectional survey studies conducted

Tracy R. G. Gladstone

tgladsto@wellesley.edu

1 The Robert S. and Grace W. Stone Primary Prevention Initiatives, Wellesley Centers for Women, Wellesley College, 106 Central Street, Wellesley, MA 02481, USA

2 University of Colorado, Boulder, CO, USA

3 Department of Counseling and Human Development, University of Louisville, Louisville, KY, USA during the pandemic have found that adolescents perceive an increase in general mental health symptoms [6], and particularly increases in feelings of unhappiness or depression [7].

The connection between negative life events and depression in youth is well established $[8,9]$, and the severity of exposure to negative life events has been associated with greater levels of depressive symptoms [10, 11]. In particular, loneliness and social isolation have been connected to depression in adolescence, especially when it is enduring [12]. As a result, Loades and colleagues [13] suggest that, in the context of the COVID-19 pandemic, current rates of depression may be particularly elevated for adolescents who are feeling socially isolated, especially as the duration of the pandemic, and the associated social isolation, extend over time. In fact, longitudinal studies of participants assessed before and then several months following the implementation of pandemic-related restrictions found that symptoms of depression increased most significantly for adolescents and/or young adults, particularly females, who reported higher levels of pandemic-related distress from, for example, not being able to see friends or having a family member or friend get very sick from COVID-19 [14]. School-related stress and school performance also have been connected to symptoms of depression in adolescents [15], and worries related to online learning and learning from home have been 
associated with increased rates of depression among adolescents during the COVID-19 pandemic [16].

Although we know that, in general, adolescents who are exposed to negative life events are more likely to experience depression [17, 18], not all adolescents who experience difficult life circumstances respond with depression. Prior research suggests that the interaction between negative life events and the way that children and adolescents generally respond to these events impacts whether or not depressive symptoms develop. Response patterns are grouped into vulnerability factors, which are associated with the increased likelihood of depressive symptoms [19,20], and protective factors, which are associated with a decrease in the likelihood of negative outcomes such as depression [21], even in the presence of significant negative life events.

Vulnerability-stress models, such as the cognitive theory of depression [22] and the hopelessness theory [23], may explain the onset of depressive symptoms in youth who experience negative life events. For example, Lewinsohn et al. [24] found that adolescents who report high levels of dysfunctional attitudes - a core vulnerability in Beck's theory [22] - are more likely to experience depressive disorders in the presence of significant negative life events than their peers reporting low or intermediate levels of dysfunctional attitudes. Likewise, Hankin [25] found support for the interaction between negative cognitive style, as proposed in the hopelessness theory, and negative life events in predicting symptoms of depression in 11 to 17 year old adolescents. Consistent with these vulnerability-stress models, the COVID-19 pandemic may be a large-scale negative life event that, in interaction with dysfunctional attitudes and a negative cognitive style, may be associated with increased levels of depressive symptoms among adolescents.

Protective factors, in contrast, may explain why adolescents who are exposed to negative life events may not develop depression. For example, resilience, a personal quality in which individuals "thrive in the face of adversity" [26], can be viewed as a protective factor that is operationalized as a set of personal qualities that enable positive responses to difficult circumstances, such as commitment, viewing challenges as opportunities, and adaptability. Given that research on resilience among children and adolescents has included multiple negative life events (e.g., poverty, chronic illness, catastrophic life events [27]), it is reasonable to consider the impact of resilience on psychological adjustment in response to the COVID-19 pandemic. However, to date, the majority of research on resilience and the pandemic has focused on adults [28]. Likewise, a supportive relationship with parents is an additional protective factor that is associated with adolescents' reduced risk for depression, even in the presence of significant negative life events. Prior research on youth depression indicates that depressive outcomes are connected to the quality of adolescents' relationships with both mothers and fathers [29], even more so than to the quality of peer relationships [30]. Moreover, Delay and colleagues [31] found that adolescents' perceptions of parental support moderated the relation between a negative life event (i.e., peer victimization) and self-reported symptoms of depression, and Hazel and colleagues [32] similarly reported a significant depression-buffering effect of parental relationship quality, whether adolescent- or parent- reported, on adolescents' depressive symptoms when confronted by peer-related negative experiences.

Research on depression in adolescent samples that have been exposed to significant negative life events, such as an earthquake or a terrorist attack, suggest that adolescents vary in their responses to those events, based on a range of vulnerability factors, such as dysfunctional attitudes and negative cognitive style, and also protective factors, such as resilience and parental support. Ye, Fan, Li and Han [33], for example, reported that adolescents exposed to the 2008 Wenchuan earthquake in China were more likely to experience depressive symptoms 6-12 months after the disaster when they were female, less resilient, and reported more significant exposure to the earthquake (i.e., a family member was injured or died, their home was damaged). Likewise, relative to those with less parental support, Israeli adolescents living in areas routinely targeted by rockets were less likely to experience symptoms of depression when they also reported high levels of social support from parents, even when their exposure to the attacks was high (e.g., they or a friend/family member were physically hurt from a rocket attack, they experienced property damage as a result of a rocket attack) [34]. Finally, a recent online study of the effects of the COVID-19 pandemic on depressive symptoms in a large sample of Chinese adolescents, surveyed once following the onset of the pandemic, revealed that both lower levels of social support and also more personal exposure to COVID-19 were associated with higher levels of depressive symptoms [35].

Although several researchers suggest that, as a large-scale negative life event, the COVID-19 pandemic will likely have significant mental health consequences on youth (e.g., Courtney et al., [2]), we are aware of only two longitudinal studies that actually explored changes in symptoms of depression across the COVID-19 pandemic for adolescents and young adults $[14,16]$. Moreover, we are not aware of any studies that have explored factors that moderate the relation between distress from COVID-19 as a significant life event and depressive symptoms among adolescents. The first aim of the current study was to examine the change in depressive symptoms in adolescents who had been screened for depression as part of a school program pre-pandemic and then were reassessed two months after the issuance of a stay-at-home order. Our second aim was to explore the possible moderating effects of vulnerability (i.e., dysfunctional 
attitudes, negative cognitive style) and protective (i.e., resilience, parent-child relationships) factors on depressive symptoms among adolescents with varying degrees of COVID-19-related distress. Specifically, in accordance with the vulnerability-stress model, we hypothesize that in the face of COVID-19 pandemic related challenges, adolescents with dysfunctional attitudes or a negative cognitive style will demonstrate greater levels of depressive symptoms than those without these vulnerability factors. Additionally, we hypothesize that more resilient adolescents, or those with stronger relationships with their parents, will develop fewer depressive symptoms in the midst of COVID-19 distress than those who do not possess these protective factors.

\section{Methods}

\section{Participants}

Participants included $5957^{\text {th }}, 9^{\text {th }}$ and $11^{\text {th }}$ grade students who participated in an in-person universal mental health screening program at their public school in a suburban Boston community from November, 2019 through January, 2020 (Time 1). Of this full sample, 228 adolescents (38\%) also completed a second online assessment in May, 2020 (Time 2). Adolescents who completed both assessments ranged in age from 12 to $18(M=14.5$ years, $S D=1.60)$, with $53 \%$ female, and $73 \%$ self-identified as White, $9 \%$ as Asian, $5 \%$ as Hispanic, $3 \%$ as Middle Eastern, less than $1 \%$ as Black or Native Hawaiian, and $9 \%$ identified as other or preferred to not answer. Forty-two percent of adolescents were in the $7^{\text {th }}$ grade, $33 \%$ were in the $9^{\text {th }}$ grade, and $25 \%$ were in the $11^{\text {th }}$ grade. Seventy-eight percent of adolescents' parents held at least a 4-year college degree.

\section{Measures}

\section{Depressive Symptoms}

The Patient Health Questionnaire-Adolescent (PHQ-A) The PHQ-A is a 9-item self-report screening measure for depression, which adolescents completed at Time 1. The PHQ-A assesses symptoms of depression over the past two weeks using a 4-point Likert scale. The PHQ-A demonstrates adequate psychometric properties $(\alpha=0.898)$. At Time 2 , adolescents completed the PHQ-A without the $9^{\text {th }}$ question assessing suicidal thinking/behavior, since the Time 2 assessment was conducted online $(\alpha=0.891)$. This version of the PHQ is recommended for use when clinicians are not available to provide immediate intervention, if indicated [36], and identical scoring thresholds are appropriate for both measures [37]. To maintain consistency across time- points, we calculated PHQ-8 scores for Time 1 data, and used these scores for all analyses.

\section{Vulnerability Factors}

Children's Cognitive Style Questionnaire (CCSQ) The CCSQ is a self-report measure of cognitive vulnerability to depression in minors [38]. The CCSQ presents four negative event scenarios and two positive life event scenarios. Each scenario is followed by five statements that determine the individual's tendency to make negative inferences about causes (global/specific, stable/unstable, and internal/external) and consequences of the event and self-characteristics. Participants respond to each statement on a 5-point Likert scale from 1 (do not agree at all) to 5 (agree a lot). This study used a 16-item version of the measure, using only the negative event scenarios and excluding the internal/external attribution about causes of the event item from each scenario, which is not relevant for depression following the hopelessness model [23]. A dimensional mean was computed using an average of three subscale means: negative inference about causes of the event score, negative consequences of the event score, negative self-inferences score. This questionnaire was given to participants at Time $2(\alpha=0.843)$.

Dysfunctional Attitude Scale (DAS-9) The DAS-9 [39] is a 9-item self-report measure that aims to determine stable dysfunctional attitudes that people with depression hold about themselves, the world, and their future. The measure uses a 5-point Likert scale from 1 (Totally agree) to 5 (Totally disagree). Participants completed this measure at Time $2(\alpha=0.828)$.

\section{Protective Factors}

Conflict Behavior Questionnaire (CBQ) The CBQ was designed for the purpose of evaluating parent and adolescent behavior directly from mother and adolescent [40]. For this study, the adolescent version only was used, and the term "mother" was replaced with "parent." The CBQ measures two sources of complaints: dissatisfaction with the other person's behavior, and evaluations of the interactions between the two family members. Participants completed this measure at Time $2(\alpha=0.919)$.

Connor-Davidson Resiliency Scale (CD-RISC) The 10-item CD-RISC 10 is a self-report scale that measures resilience against adversity [41]. The measure uses a 5-point Likert scale from 0 (not true at all) to 4 (true nearly all the time) to determine resilience, with a total possible score of 40 indicating the highest level of resilience. This scale has adequate psychometric properties $(\alpha=0.850)$ and was given to participants at Time 2 . 


\section{Covid-19-Related Distress}

A questionnaire was developed for this study containing 39 items regarding the impact of the COVID-19/Coronavirus pandemic on adolescents. The questions assess mood, coping skills, exposure to illness, and changes to daily life. The measure uses a 5-point Likert scale to assess how much the respondent has been affected by each item. Sample items include "How much have you been practicing quarantine guidelines," "How much have you had trouble completing all of your homework," and "How often have you spent time outdoors."

From this questionnaire, 30 items used the same 5 -point response scale ranging from 1 (None/Never) to 5 (A lot/ Always). These 30 items were entered into an exploratory factor analysis, which suggested the items loaded on 8 separate factors. For the purposes of this paper, we focused on the first factor, which included many of the items we would theoretically expect to be related to COVID-19-related distress, based on prior studies indicating the aspects of the pandemic that adolescents view as most distressing (e.g., Hawes et al. [16], Loades et al., [13], Magson et al., [14]). We retained all of the items in this first factor with factor loadings greater than 0.5 , resulting in 14 items. Another item "How much have your responsibilities at home changed?" was also included in this scale due to its theoretical importance, even though it had a factor loading of less than 0.5. To avoid any overlaps with our outcome measure, we removed 4 items related to depression symptoms ("felt more sad/ down/ depressed than usual?", "felt more stressed/overwhelmed/ anxious than usual?", "slept for more/less time than usual?" and "eaten more/less than usual?"), one item related to mood changes ("How much has your overall mood changed?") and two items related to coping behaviors ("tried to distract yourself" and "chosen to spend time alone"). Our final COVID-19-related distress scale consists of 8 items $(\alpha=0.806)$, including: "How much have you thought about the COVID-19/Coronavirus pandemic," "How much have you thought about whether people would get sick with COVID-19/Coronavirus," "In general, how worried are you about the COVID-19/Coronavirus pandemic," "How much have you felt more lonely/ isolated than usual," "How much has your daily life been impacted," "How much have your responsibilities at home changed," "How much have you had trouble completing all of your homework," and "How often have you argued/had conflict with your friends or family."

\section{Procedure}

At Time 1, data were collected in-person during class time with paper-and-pencil questionnaires, as part of an in-school mental health screening of the entire $7^{\text {th }}, 9^{\text {th }}$ and $11^{\text {th }}$ grades.
The participation rate in the Time 1 screening was 93\%; parents were given the option prior to the screening to opt out their children, and some children were absent on multiple screening days or decided themselves that they did not want to participate. At Time 2, all data were collected online through a secure web-based system (Qualtrics), which students accessed from home via personal computers during the early stages of the Covid-19 lockdown. Parents received a letter from the school via email describing the Time 2 data collection and inviting them to opt-out their children. Thereafter, adolescents received questionnaires via their school's online student software, and all adolescents provided electronic assent prior to completing the Time 2 questionnaires. Adolescents received a small gift card upon completing the Time 2 assessment. The study was approved by the BLINDED Institutional Review Board.

\section{Data Analysis}

To test whether there were any differences between the sample of students who completed both data collections and those who only completed the measures at Time 1, independent samples t-tests were used to compare depression scores, and chi-square analyses were used to test demographic differences. Regarding our first aim, paired samples $\mathrm{t}$-tests were used to explore differences in depression scores for students who completed both time points.

Regarding our second aim, correlation analyses were used to test the relationships between the independent variables and Time 2 depression scores. Individual linear regression models were conducted to test the associations between each of the independent variables and Time 2 depression scores. The next set of linear regressions included COVID19-related distress as another predictor. Lastly, individual interaction terms were created between COVID-19-related distress and the risk (CCSQ and DAS-9) and protective (CBQ, CD-RISC) factors to test the combined effects of COVID-19-related distress and the predictor on Time 2 depression scores. All regression models controlled for Time 1 depression scores and student gender. Students reporting a non-binary gender $(\mathrm{N}=6)$ were excluded from the model and treated as missing data. Analyses were conducted using Statistical Package for the Social Sciences (SPSS) version 26 and missing data was handled using listwise deletion.

\section{Results}

There were no differences in Time 1 PHQ-A scores between adolescents who completed the measure at both time points $(\mathrm{n}=228)$ and adolescents who completed the measure at Time 1 only $(\mathrm{n}=355)(t(581)=0.23, p=0.819)$. Chi-square analysis found that the subsample of adolescents who 
completed assessments at both time points included a greater percentage of females $\left(x^{2}(1, n=569)=13.33, p<0.001\right)$ and 7 th graders $\left(x^{2}(2, n=583)=6.36, p=0.042\right)$.

Clinically, overall the sample of adolescents reported minimal depressive symptoms at both Time $1(M=3.44$, $S D=4.55)$ and Time $2(M=4.37, S D=4.88)$. Specifically, at Time $1,1 \%$ of adolescents reported depressive symptoms in the severe range, $3.9 \%$ in the moderately severe range, $4.5 \%$ in the moderate range, $14.9 \%$ in the mild range, and $75.6 \%$ in the none to minimal range. At Time 2, $1.8 \%$ of adolescents reported depressive symptoms in the severe range, $3.9 \%$ in the moderate to severe range, $9.6 \%$ in the moderate range, $22.8 \%$ in the mild range, and $61.8 \%$ in the none to minimal range.

Regarding our first aim, Time 2 PHQ-A scores were significantly higher than Time 1 scores $(t(227)=3.56$, $p<0.001)$. In particular, depressive symptoms were significantly higher at Time 2 compared to Time 1 for female adolescents $(t(120)=-3.55, p=0.001)$.

Regarding our second aim, correlational analyses were conducted to explore the associations between the CCSQ, DAS-9, CBQ, CD-RISC, COVID-19-related distress, and depression scores at Time 2. All predictors were significantly related to depression scores at Time 2 (Table 1).

The initial set of linear regression models assessed the individual effects of the independent variables on depression scores at Time 2, controlling for gender and Time 1 depression scores. Higher resilience $(B=-0.26, S E=0.04$, $\mathrm{p}<0.001)$ and more functional attitudes $(\mathrm{B}=-1.72$, $\mathrm{SE}=0.32, \mathrm{p}<0.001)$ were significantly related to lower Time 2 depression scores. Less adaptive cognitive styles $(\mathrm{B}=3.27, \mathrm{SE}=0.37, \mathrm{p}<0.001)$ and more parental conflict $(\mathrm{B}=0.20, \mathrm{SE}=0.07, \mathrm{p}=0.003)$ were related to higher depression scores at Time 2 (see Table 2).

The next set of models included both the predictors and COVID-19-related distress. For resilience, higher resilience $(\mathrm{B}=-0.18, \mathrm{SE}=0.03, \mathrm{p}<0.001)$ was associated with lower depression scores, but higher COVID-19-related distress $(\mathrm{B}=2.25, \mathrm{SE}=0.29, \mathrm{p}<0.001)$ was associated with higher depression scores. For the DAS-9, fewer dysfunctional attitudes $(\mathrm{B}=-1.16, \mathrm{SE}=0.29, \mathrm{p}<0.001)$ were associated with lower Time 2 depression scores, and higher COVID-19-related distress $(\mathrm{B}=2.43, \mathrm{SE}=0.30, \mathrm{p}<0.001)$ was associated with higher depression scores. For the CCSQ, both higher negative cognitive styles $(\mathrm{B}=2.02, \mathrm{SE}=0.36, \mathrm{p}<0.001)$ and higher COVID-19-related distress $(\mathrm{B}=2.00, \mathrm{SE}=0.31$, $\mathrm{p}<0.001)$ were associated with higher depression scores at
Time 2. Finally, parental conflict on the CBQ was not significantly associated with Time 2 depression scores when COVID-19 related distress was entered into the model, but COVID-19-related distress $(\mathrm{B}=2.65, \mathrm{SE}=0.31, \mathrm{p}<0.001)$ was associated with higher Time 2 depression scores (see Table 2).

The last set of regression models created individual interaction terms to test the combined effects of COVID-19-related distress and the predictor on Time 2 depression scores. Significant interactions were found between COVID-19-related distress and resilience on the CD-RISC $(B=-0.13$, $\mathrm{SE}=0.03, \mathrm{p}<0.001)$ and COVID-19-related distress and negative cognitive styles on the CCSQ $(B=1.29, \mathrm{SE}=0.33$, $\mathrm{p}<0.001$ ) (see Table 2). Specifically, high COVID-19-related distress and low resilience was associated with high depression scores, while low COVID-19-related distress and high resilience was associated with lower depression scores at Time 2 (Fig. 1). In addition, high COVID-19-related distress and high negative cognitive styles were associated with high depression scores, while low COVID-19-related distress and low negative cognitive styles was associated with lower depression scores at Time 2 (Fig. 2).

\section{Discussion}

We were provided with a unique opportunity to examine the effects of a large-scale negative life event, the COVID-19 pandemic, on depressive symptoms, and also to examine moderating effects of vulnerability and protective factors, in a school-based group of adolescents. While depressive symptoms were significantly higher when assessed during the pandemic compared to before, the overall mean PHQ-A score fell in the minimal symptom range of the measure at both timepoints. This suggests that, although adolescents as a whole are experiencing a greater level of distress while being exposed to this negative life event, for many adolescents this distress represents a normative reaction and not one that rises to the level of a disorder [42]. While research focusing on the pandemic reveals greater increases in depression in adult samples during this time of stress [43], it is important to note that the moderate increases in depressive symptoms reported here are consistent with similar studies of youth during the first months of the pandemic [14, 16]. It will be important to see if more clinically relevant levels of depression are more common as pandemic restrictions continue.

Table 1 Correlation results

\begin{tabular}{llllll}
\hline & COVID-19 related distress & DAS-9 & CCSQ & CD-RISC & CBQ \\
\hline Depression Scores (Time 2) & $.604 * * *$ & $-.448^{* * *}$ & $.613^{* * *}$ & $-.556^{* * *}$ & $.338^{* * * *}$ \\
\hline$* * * p<.001$ & & & & &
\end{tabular}


Table 2 Regression results

\begin{tabular}{|c|c|c|c|c|c|c|c|c|c|c|c|c|}
\hline & \multicolumn{4}{|c|}{ Step 1 Model: Predictor Only } & \multicolumn{4}{|c|}{$\begin{array}{l}\text { Step } 2 \text { Model: COVID Distress \& } \\
\text { Predictor }\end{array}$} & \multicolumn{4}{|c|}{ Step 3 Model: Interaction } \\
\hline & \multicolumn{4}{|l|}{$\mathrm{N}=201$} & \multicolumn{4}{|l|}{$\mathrm{N}=197$} & \multicolumn{4}{|l|}{$\mathrm{N}=197$} \\
\hline & B & SE & $\beta$ & $\mathrm{p}$ & B & SE & $\beta$ & $\mathrm{p}$ & B & SE & $\beta$ & $\mathrm{p}$ \\
\hline COVID distress & & & & & 2.25 & 0.29 & 0.39 & $<.001$ & 5.39 & 0.79 & 0.95 & $<.001$ \\
\hline Resilience scale & -0.26 & 0.04 & -0.39 & $<.001$ & -0.18 & 0.03 & -0.27 & $<.001$ & 0.21 & 0.10 & 0.31 & 0.03 \\
\hline Resilience X COVID & & & & & & & & & -0.13 & 0.03 & -0.66 & $<.001$ \\
\hline Depression (T1) & 0.45 & 0.06 & 0.43 & $<.001$ & 0.38 & 0.05 & 0.36 & $<.001$ & 0.38 & 0.05 & 0.37 & $<.001$ \\
\hline Gender & 0.76 & 0.47 & 0.09 & 0.11 & 0.20 & 0.43 & 0.02 & 0.64 & 0.10 & 0.41 & 0.01 & 0.804 \\
\hline R-square & 0.461 & & & & 0.590 & & & & 0.625 & & & \\
\hline \multirow[t]{3}{*}{$\Delta$ in $\mathrm{R}$-square } & & & & & 0.129 & & & & 0.035 & & & \\
\hline & \multicolumn{4}{|l|}{$\mathrm{N}=204$} & \multicolumn{4}{|l|}{$\mathrm{N}=197$} & \multicolumn{4}{|l|}{$\mathrm{N}=197$} \\
\hline & B & SE & $\beta$ & $\mathrm{p}$ & $\mathrm{B}$ & $\mathrm{SE}$ & $\beta$ & $\mathrm{p}$ & B & SE & $\beta$ & $\mathrm{p}$ \\
\hline COVID distress & & & & & 2.43 & 0.30 & 0.43 & $<.001$ & 4.53 & 1.16 & 0.80 & $<.001$ \\
\hline DAS-9 scale & -1.72 & 0.32 & -0.30 & $<.001$ & -1.16 & 0.29 & -0.20 & $<.001$ & 0.40 & 0.88 & 0.07 & 0.65 \\
\hline DAS-9 X COVID & & & & & & & & & -0.57 & 0.31 & -0.40 & 0.06 \\
\hline Depression (T1) & 0.47 & 0.06 & 0.45 & $<.001$ & 0.39 & 0.05 & 0.37 & $<.001$ & 0.38 & 0.05 & 0.37 & $<.001$ \\
\hline Gender & 1.36 & 0.48 & 0.16 & 0.005 & 0.59 & 0.44 & 0.07 & 0.18 & 0.55 & 0.44 & 0.06 & 0.21 \\
\hline R-square & 0.414 & & & & 0.565 & & & & 0.573 & & & \\
\hline \multirow[t]{3}{*}{$\Delta$ in R-square } & & & & & 0.151 & & & & 0.008 & & & \\
\hline & \multicolumn{4}{|l|}{$\mathrm{N}=214$} & \multicolumn{4}{|l|}{$\mathrm{N}=197$} & \multicolumn{4}{|l|}{$\mathrm{N}=197$} \\
\hline & B & SE & $\beta$ & $\mathrm{p}$ & $\mathrm{B}$ & $\mathrm{SE}$ & $\beta$ & $\mathrm{p}$ & B & SE & $\beta$ & $\mathrm{p}$ \\
\hline COVID distress & & & & & 2.00 & 0.31 & 0.35 & $<.001$ & -0.24 & 0.65 & -0.04 & 0.71 \\
\hline CCSQ scale & 3.27 & 0.37 & 0.46 & $<.001$ & 2.02 & 0.36 & 0.30 & $<.001$ & -2.40 & 1.18 & -0.36 & 0.04 \\
\hline CCSQ X COVID & & & & & & & & & 1.29 & 0.33 & 0.92 & $<.001$ \\
\hline Depression (T1) & 0.43 & 0.06 & 0.39 & $<.001$ & 0.37 & 0.05 & 0.35 & $<.001$ & 0.41 & 0.05 & 0.39 & $<.001$ \\
\hline Gender & 0.71 & 0.45 & 0.08 & 0.12 & 0.39 & 0.42 & 0.04 & 0.36 & 0.37 & 0.41 & 0.04 & 0.36 \\
\hline R-square & 0.514 & & & & 0.595 & & & & 0.625 & & & \\
\hline \multirow[t]{3}{*}{$\Delta$ in R-square } & & & & & 0.081 & & & & 0.030 & & & \\
\hline & \multicolumn{4}{|l|}{$\mathrm{N}=196$} & \multicolumn{4}{|l|}{$\mathrm{N}=196$} & \multicolumn{4}{|l|}{$\mathrm{N}=196$} \\
\hline & B & SE & $\beta$ & $\mathrm{p}$ & B & SE & $\beta$ & $\mathrm{p}$ & B & SE & $\beta$ & $\mathrm{p}$ \\
\hline COVID distress & & & & & 2.65 & 0.31 & 0.46 & $<.001$ & 2.23 & 0.38 & 0.39 & $<.001$ \\
\hline CBQ scale & 0.20 & 0.07 & 0.18 & 0.003 & 0.08 & 0.06 & 0.07 & 0.18 & -0.25 & 0.18 & -0.23 & 0.17 \\
\hline CBQ X COVID & & & & & & & & & 0.10 & 0.06 & 0.34 & 0.06 \\
\hline Depression (T1) & 0.50 & 0.06 & 0.49 & $<.001$ & 0.41 & 0.05 & 0.40 & $<.001$ & 0.42 & 0.05 & 0.41 & $<.001$ \\
\hline Gender & 1.50 & 0.52 & 0.17 & 0.004 & 0.57 & 0.46 & 0.06 & 0.21 & 0.54 & 0.45 & 0.06 & 0.24 \\
\hline R-square & 0.360 & & & & 0.539 & & & & 0.548 & & & \\
\hline$\Delta$ in R-square & & & & & 0.179 & & & & 0.009 & & & \\
\hline
\end{tabular}

Female adolescents reported significantly more depressive symptoms during, compared to before, the pandemic. For female adolescents only, it is notable that the mean depression score fell in the mild to moderate symptom range at Time 2 . The increased depression scores in female adolescents during the pandemic is consistent with prior research that suggests adolescent girls are at higher risk of experiencing mental health symptoms in response to adverse life events [44] and findings that suggest female gender is associated with higher levels of depressive symptoms during the COVID pandemic [14, 45, 46].

In terms of the moderating effects of vulnerability and protective factors, our results suggest that, after controlling for gender and Time 1 depression, negative cognitive style and resilience moderated the relation between COVID-19-related distress and changes in depression. As we predicted, the tendency to draw more negative inferences about the causes and consequences of negative 
Fig. 1 COVID-19 related related distress by resilience (CD-RISC)
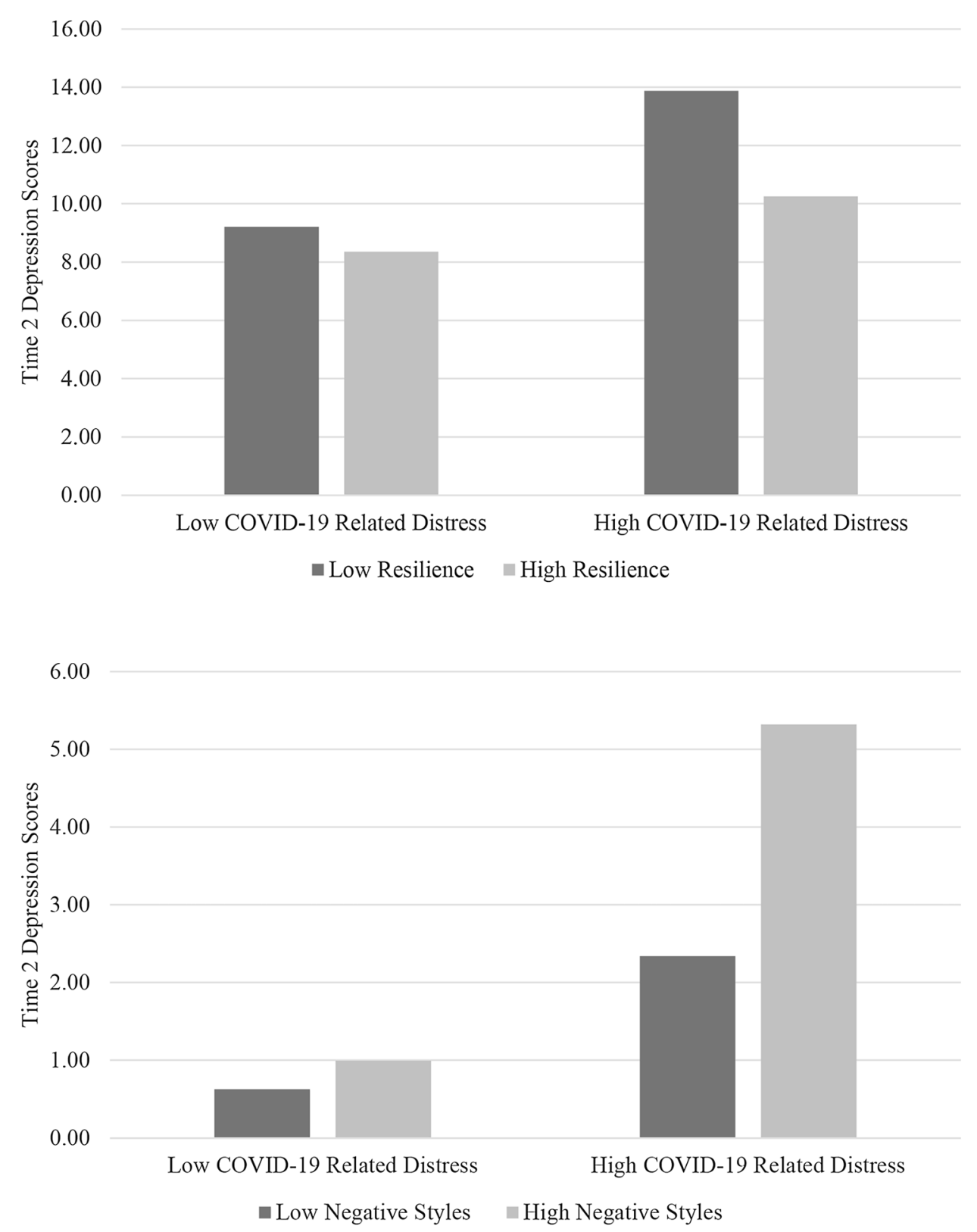

Fig. 2 COVID-19 related related distress by negative cognitive style (CCSQ) events and self-characteristics served as a vulnerability factor for depressive symptoms. Among students who were more distressed about the effects of the pandemic, it was those with a more negative cognitive style who showed greater increases in depression relative to those with a less negative cognitive style. Also as predicted, resilience served as a protective factor. Adolescents who reported greater resilience showed less of an increase in depression than less resilient teens when confronted with distress about COVID-19. These results are consistent with the vulnerability-stress and protective models. Further, they suggest that the risk for developing depressive symptoms in response to distress caused by negative life events depends on individual vulnerability and protective factors.
While the other vulnerability (i.e., dysfunctional attitudes) and protective (i.e., relationships with parents) factors explored in this study were not found to significantly moderate the relations between COVID-19 distress and changes in depression, they both demonstrated trends in the expected direction. While, theoretically, Beck's [22] and Abramson's [23] theories make similar predictions regarding unique cognitive vulnerability factors for depression, it is not uncommon for data among adolescents to support one model over the other $[24,47]$. We made no a priori hypotheses about differences between cognitive style versus dysfunctional attitudes and suggest that future studies may want to explore nuances with respect to how different cognitive styles interact with the challenges of particular negative life 
events (such as COVID-19) in relation to depression. Our finding that the interaction between relations with parents and COVID-19 distress did not significantly predict changes in depression is somewhat surprising given the previous literature on the moderating effects of parent-child relations and negative events in predicting depression among youth [32]. However, it may be that in the context of the pandemic, the importance of interactions with friends (or lack thereof), something we did not measure in this study, is especially pronounced for adolescents [14].

The current study is not without limitations. First, our response rate at Time 2 was 38\%, which is not surprising given the challenges associated with online data collection [48], the only option available during the COVID-19 pandemic. In fact, the response rate reported here is above the $<25 \%$ response rate reported for a study of adolescents in New Orleans, LA who, after completing questionnaires in class about their experiences prior to the pandemic, were asked to complete fully online questionnaires about their experiences during the pandemic [49]. Response rates are a consistent problem for online data collection [48], but the connection between response rate and study validity has been found to be weak $[50,51]$. While it is possible that this low response rate biased our sample, analyses suggest that the subsample of students with data at two time points had similar baseline scores on the PHQ- 8 compared to the students who completed questionnaires only at the first timepoint. Thus, the students who completed both timepoints appear to be comparable to those who completed a single timepoint clinically. Moreover, the depression rates at Time 2 from our study are not only similar to the rates obtained at Time 1, but are also consistent with depression levels found in other studies of adolescents and young adults during the first part of the pandemic $[14,16]$.

Second, participants were recruited from a single suburban school. As such, the sample was demographically restricted, and our participants were predominately White and from highly educated families. The lack of diversity in our sample may affect generalizability of our findings. Third, while we believe the longitudinal nature of our study is a strength, measures of dysfunctional attitudes, negative cognitive styles, parent support, and resilience were measured only at Time 2 . While each of these measures was designed to measure stable traits as demonstrated by their adequate to good test-retest reliability scores [26, 38, 52-54], it is not possible to determine causal relations between these factors and changes in depression. It is possible, for example, that increases in adolescents' depressive symptoms may bias their self-reports of resilience and cognitive style.

Finally, it is important to note that these findings only pertain to adolescents' experiences of depressive symptoms in the months directly following the start of the pandemic; it is likely that, as the pandemic persists, adolescents' responses to this event will shift over time [2]. In fact, consistent with the findings reported here, Magson and colleagues [14] reported an increase in depressive symptoms among adolescents shortly following the start of the pandemic. Likewise, in a college age sample, Charles and colleagues [55] found an initial increase in depressive symptoms in the spring of 2020 , but they found that symptom scores had returned to pre-pandemic levels by the fall of 2020. In contrast, Luthar and colleagues [56] reported that depressive symptoms in an adolescent sample fell in the weeks just following the issuance of stay-at-home orders, which they attribute to the reduced stress adolescents experienced as they had fewer activities and academic pressures. Unfortunately, data on the longer-term effects of the Covid-19 pandemic on depressive symptoms in youth are not currently available, but it will be important to examine the trajectory of adolescents' depressive symptoms over time, as lockdown restrictions are lifted and daily activities resume.

Despite these limitations, our findings contribute to the evidence beginning to emerge about the mental health of adolescents during the first part of the COVID-19 pandemic, with implications for our understanding of how adolescents respond to significant negative life events more generally. Specifically, like other researchers, we found elevated levels of depressive symptoms among teens during the first few months of the pandemic compared to pre-pandemic levels. These increases in depression were especially pronounced among girls. In addition, teens with negative cognitive style, and adolescents who felt more distressed by COVID-19 and the impact it was having on their lives, showed greater increases in depression. In contrast, those youth with greater resiliency and better relationships with their parents experienced less of an increase in depression during the first few months of the pandemic. Unique to this study, we explored vulnerability and protective factors that might moderate the relation between COVID-19-related distress and changes in depression. We found support for the vulnerability-stress model, such that negative cognitive style in interaction with COVID-19-related distress was associated with increased depression. We also found support for the protective factors theory, such that, compared to students with less resilience, those who reported greater personal resilience showed less of an increase in depression when COVID-19-related distress was high.

Findings from this research address the potential implications of significant negative life events on adolescents' depression risk over time. In fact, given that adolescent depression has long-term consequences [57], and that negative life events experienced by adolescents predict risk for depression during adulthood [58], it is important for mental health professionals and school personnel to be aware of the potential increases in depression that may emerge for adolescents during the pandemic. It is certainly possible that, 
broadly speaking, adolescents who have lived through this large-scale negative life event will experience higher levels of depression throughout adulthood, with significant implications for their long-term health, and for the health and well-being of many in their generation.

In addition, these findings suggest the importance of exploring ways to decrease vulnerability factors while strengthening protective factors in order to support adolescents who may be experiencing distress related to significant negative life events, in order to diminish the impact of those events. It is also vital that researchers and professionals continue to assess and monitor students over time, as it is not clear how depressive symptoms, and their relation to vulnerability and protective factors, may change as the pandemic and its associated restrictions linger and present new challenges. Finally, it will be interesting for future research to consider more integrative approaches to understanding how vulnerability and protective factors are theoretically meaningfully related to each other, in order to understand how to best help youth who develop depression during times of large-scale negative life events, and how to prevent increases in depressive symptoms altogether.

\section{Summary}

The main objective of this research was to investigate the impact of the COVID-19 pandemic on depressive symptoms among a community sample of adolescents, and to examine the relation between COVID-19-related distress and vulnerability/protective factors in accounting for change in depressive symptoms over time. The COVID-19 pandemic is a significant stressor for many adolescents, yet to date, there is limited longitudinal research examining the effect of the pandemic on depressive symptoms in adolescents, and no research examining the possible moderating effects of vulnerability (i.e., dysfunctional attitudes, negative cognitive style) and protective (i.e., resilience, strong parent/ child relationship) factors on depressive symptoms among adolescents with varying degrees of distress related to the COVID-19 pandemic. As predicted, adolescents reported an increase in symptoms of depression from before to during the pandemic, particularly among females. Moreover, as predicted, in the face of high COVID-19-related distress, low resilience and negative cognitive styles were associated with higher depression scores during the pandemic, consistent with vulnerability-stress models of depression. These results suggest that mental health professionals and school personnel should be aware of potential increases in depression among teenagers during the pandemic. In addition, these findings suggest the importance of exploring ways to decrease vulnerability factors while strengthening protective factors in order to support adolescents who may be experiencing distress related to the pandemic and other significant negative life events.

Acknowledgements Thank you to Amy Kane, M.A., L.M.H.C., Erica Plunkett, M.S. Ed., Laura Tichner, M.S.W., and the students and families from a local school district.

Funding Initial data collection for this study was supported by funds from a local school district, and follow-up data collection and analysis were supported by the Wellesley College Primary Prevention Fund.

\section{Declarations}

Conflict of Interest The authors declare that they have no conflict of interest.

Ethical approval All procedures performed in studies involving human participants were in accordance with the ethical standards of the institutional review board at BLINDED and with the 1964 Helsinki declaration and its later amendments or comparable ethical standards. The authors declare that they have no conflict of interest.

\section{References}

1. Thapar A, Collishaw S, Pine DS, Thapar AK (2012) Depression in adolescence. Lancet 379(9820):1056-1067. https://doi.org/10. 1016/S0140-6736(11)60871-4

2. Courtney D, Watson P, Battaglia M, Mulsant BH, Szatmari P (2020) COVID-19 impacts on child and youth anxiety and depression: challenges and opportunities. Can J Psychiatry 65(10):688691. https://doi.org/10.1177/0706743720935646

3. Ellis WE, Dumas TM, Forbes LM (2020) Physically isolated but socially connected: psychological adjustment and stress among adolescents during the initial COVID-19 crisis. Can J Behav Sci 52(3):177. https://doi.org/10.1037/cbs0000215

4. Gruber J, Prinstein MJ, Clark LA, Rottenberg J, Abramowitz JS, Albano AM et al (2020) Mental health and clinical psychological science in the time of COVID-19: challenges, opportunities, and a call to action. Am Psychol. https://doi.org/10.1037/amp0000707

5. Guessoum SB, Lachal J, Radjack R, Carretier E, Minassian S, Benoit $\mathrm{L}$ et al (2020) Adolescent psychiatric disorders during the COVID-19 pandemic and lockdown. Psychiatry Res 291:113264. https://doi.org/10.1016/j.psychres.2020.113264

6. Hawke LD, Barbic SP, Voineskos A, Szatmari P, Cleverley K, Hayes E et al (2020) Impacts of COVID-19 on Youth Mental Health, Substance Use, and Well-being: A Rapid Survey of Clinical and Community Samples: Répercussions de la COVID-19 sur la santé mentale, l'utilisation de substances et le bien-être des adolescents: un sondage rapide d'échantillons cliniques et communautaires. Can J Psychiatry 65(10):701-709. https://doi.org/ 10.1177/0706743720940562

7. Margolius M, Doyle Lynch A, Pufall Jones E, Hynes, M (2020) The State of Young People during COVID-19: findings from a Nationally Representative Survey of High School Youth. America's Promise Alliance. https://files.eric.ed.gov/fulltext/ED606305. pdf

8. Franko DL, Striegel-Moore RH, Brown KM, Barton BA, McMahon RP, Schreiber GB et al (2004) Expanding our understanding of the relationship between negative life events and depressive 
symptoms in black and white adolescent girls. Psychol Med 34(7):1319-1330. https://doi.org/10.1017/S0033291704003186

9. Sawyer MG, Pfeiffer S, Spence SH (2009) Life events, coping and depressive symptoms among young adolescents: a one-year prospective study. J Affect Disord 117(1-2):48-54. https://doi. org/10.1016/j.jad.2008.12.013

10. Goldmann E, Galea S (2014) Mental health consequences of disasters. Annu Rev Public Health 35:169-183. https://doi.org/10. 1146/annurev-publhealth-032013-182435

11. Zhou X, Wu X, An Y (2016) Understanding the relationship between trauma exposure and depression among adolescents after earthquake: the roles of fear and resilience. Front Psychol 7:2044. https://doi.org/10.3389/fpsyg.2016.02044

12. Qualter P, Brown SL, Munn P, Rotenberg KJ (2010) Childhood loneliness as a predictor of adolescent depressive symptoms: an 8-year longitudinal study. Eur Child Adolesc Psychiatry 19(6):493-501. https://doi.org/10.1007/s00787-009-0059-y

13. Loades ME, Chatburn E, Higson-Sweeney N, Reynolds S, Shafran R, Brigden A et al (2020) Rapid systematic review: the impact of social isolation and loneliness on the mental health of children and adolescents in the context of COVID-19. J Am Acad Child Adolesc Psychiatry 59(11):1218-1239. https://doi.org/10.1016/j. jaac.2020.05.009

14. Magson NR, Freeman JY, Rapee RM, Richardson CE, Oar EL, Fardouly J (2021) Risk and protective factors for prospective changes in adolescent mental health during the COVID-19 pandemic. J Youth Adolesc 50(1):44-57. https://doi.org/10.1007/ s10964-020-01332-9

15. Anniko M, Boersma K, Tillfors M (2019) Sources of stress and worry in the development of stress-related mental health problems: a longitudinal investigation from early-to mid-adolescence. Anxiety Stress Coping 32(2):155-167. https://doi.org/10.1080/ 10615806.2018.1549657

16. Hawes MT, Szenczy AK, Klein DN, Hajcak G, Nelson BD (2021) Increases in depression and anxiety symptoms in adolescents and young adults during the COVID-19 pandemic. Psychol Med. https://doi.org/10.1017/S0033291720005358

17. Grant KE, Compas BE, Thurm AE, McMahon SD, Gipson PY (2004) Stressors and child and adolescent psychopathology: measurement issues and prospective effects. J Clin Child Adolesc Psychol 33(2):412-425. https://doi.org/10.1207/s15374424jccp3302_ 23

18. Jenness JL, Peverill M, King KM, Hankin BL, McLaughlin KA (2019) Dynamic associations between stressful life events and adolescent internalizing psychopathology in a multiwave longitudinal study. J Abnorm Psychol 128(6):596-609. https://doi.org/ $10.1037 / a b n 0000450$

19. Hankin BL, Abramson LY, Miller N, Haeffel GJ (2004) Cognitive vulnerability-stress theories of depression: examining affective specificity in the prediction of depression versus anxiety in three prospective studies. Cog Ther Res 28(3):309-345. https://doi.org/ 10.1023/B:COTR.0000031805.60529.0d

20. Jacobs RH, Reinecke MA, Gollan JK, Kane P (2008) Empirical evidence of cognitive vulnerability for depression among children and adolescents: a cognitive science and developmental perspective. Clin Psychol Rev 28(5):759-782. https://doi.org/10.1016/j. cpr.2007.10.006

21. Kazdin AE, Kraemer HC, Kessler RC, Kupfer DJ, Offord DR (1997) Contributions of risk-factor research to developmental psychopathology. Clin Psychol Rev 17(4):375-406. https://doi. org/10.1016/S0272-7358(97)00012-3

22. Beck AT (1976) Cognitive therapy and the emotional disorders. International Universities Press, New York

23. Abramson LY, Metalsky GI, Alloy LB (1989) Hopelessness depression: a theory-based subtype of depression. Psychol Rev 96(2):358-372. https://doi.org/10.1037/0033-295X.96.2.358
24. Lewinsohn PM, Joiner TE Jr, Rohde P (2001) Evaluation of cognitive diathesis-stress models in predicting major depressive disorder in adolescents. J Abnorm Psychol 110(2):203-215. https:// doi.org/10.1037/0021-843X.110.2.203

25. Hankin B (2008) Cognitive vulnerability-stress model of depression during adolescence: investigating depressive symptom specificity in a multi-wave prospective study. J Abnorm Child Psychol 36(7):999-1014. https://doi.org/10.1007/s10802-008-9228-6

26. Connor KM, Davidson JRT (2003) Development of a new resilience scale: The connor-davidson resilience scale (CD-RISC). Depress Anxiety 18(2):76-82. https://doi.org/10.1002/da.10113

27. Luthar SS, Cicchetti D, Becker B (2000) The construct of resilience: a critical evaluation and guidelines for future work. Child Dev 71(3):543-562. https://doi.org/10.1111/1467-8624.00164

28. Killgore WD, Taylor EC, Cloonan SA, Dailey NS (2020) Psychological resilience during the COVID-19 lockdown. Psychiatry Res 291:113216. https://doi.org/10.1016/j.psychres.2020.113216

29. Sheeber LB, Davis B, Leve C, Hops H, Tildesley E (2007) Adolescents' relationships with their mothers and fathers: associations with depressive disorder and subdiagnostic symptomatology. J Abnorm Psychol 116(1):144-154. https://doi.org/10.1037/0021843X.116.1.144

30. Stice E, Ragan J, Randall P (2004) Prospective relations between social support and depression: Differential direction of effects for parent and peer support? J Abnorm Psychol 113(1):155-159. https://doi.org/10.1037/0021-843X.113.1.155

31. DeLay D, Hafen CA, Cunha JM, Weber LN, Laursen B (2013) Perceptions of parental support buffer against depression for Brazilian youth with interpersonal difficulties. Int J Behavl Dev 37(1):29-34. https://doi.org/10.1177/0165025412454031

32. Hazel NA, Oppenheimer CW, Technow JR, Young JF, Hankin BL (2014) Parent relationship quality buffers against the effect of peer stressors on depressive symptoms from middle childhood to adolescence. Dev Psychol 50(8):2115-2123. https://doi.org/10. 1037/a0037192

33. Ye Y, Fan F, Li L, Han Q (2014) Trajectory and predictors of depressive symptoms among adolescent survivors following the Wenchuan earthquake in China: a cohort study. Soc Psychiatry Psychiatr Epidemiol 49(6):943-952. https://doi.org/10.1007/ s00127-014-0821-4

34. Shahar G, Henrich CC (2016) Perceived family social support buffers against the effects of exposure to rocket attacks on adolescent depression, aggression, and severe violence. J Fam Psychol 30(1):163-168. https://doi.org/10.1037/fam0000179

35. Qi M, Zhou SJ, Guo ZC, Zhang LG, Min HJ, Li XM et al (2020) The effect of social support on mental health in Chinese adolescents during the outbreak of COVID-19. J Adolesc Health 67(4):514-518. https://doi.org/10.1016/j.jadohealth.2020.07.001

36. Kroenke K, Strine TW, Spitzer RL, Williams JB, Berry JT, Mokdad AH (2009) The PHQ-8 as a measure of current depression in the general population. J Affect Disord 114(1-3):163-173. https:// doi.org/10.1016/j.jad.2008.06.026

37. Kroenke K, Spitzer RL (2002) The PHQ-9: a new depression diagnostic and severity measure. Psychiatr Ann 32(9):509-515. https://doi.org/10.3928/0048-5713-20020901-06

38. Mezulis AH, Hyde JS, Abramson LY (2006) The developmental origins of cognitive vulnerability to depression: temperament, parenting, and negative life events in childhood as contributors to negative cognitive style. Dev Psychol 42(6):1012-1025. https:// doi.org/10.1037/0012-1649.42.6.1012

39. Lewinsohn PM, Roberts RE, Seeley JR, Rohde P, Gotlib IH, Hops H (1994) Adolescent psychopathology: II. Psychosocial risk factors for depression. J Abnorm Psychol 103(2):302-315. https:// doi.org/10.1037/0021-843X.103.2.302

40. Prinz RJ, Foster S, Kent RN, O’Leary KD (1979) Multivariate assessment of conflict in distressed and nondistressed 
mother-adolescent dyads. J Appl Behav Anal 12(4):691-700. https://doi.org/10.1901/jaba.1979.12-691

41. Campbell-Sills L, Stein MB (2007) Psychometric analysis and refinement of the connor-davidson resilience scale (CD-RISC): Validation of a 10-item measure of resilience. J Trauma Stres 20(6):1019-1028. https://doi.org/10.1002/jts.20271

42. Galatzer-Levy IR, Huang SH, Bonanno GA (2018) Trajectories of resilience and dysfunction following potential trauma: a review and statistical evaluation. Clin Psychol Rev 63:41-55. https://doi. org/10.1016/j.cpr.2018.05.008

43. Ettman CK, Abdalla SM, Cohen GH, Sampson L, Vivier PM, Galea S (2020) Prevalence of depression symptoms in US adults before and during the COVID-19 pandemic. JAMA Netw Open 3(9):e2019686-e2019686. https://doi.org/10.1001/jamanetwor kopen.2020.19686

44. McLaughlin KA, Koenen KC, Hill ED, Petukhova M, Sampson NA, Zaslavsky AM et al (2013) Trauma exposure and posttraumatic stress disorder in a national sample of adolescents. J Am Acad Child Adolesc Psychiatry 52(8):815-830. https://doi.org/10. 1016/j.jaac.2013.05.011

45. Chen F, Zheng D, Liu J, Gong Y, Guan Z, Lou D (2020) Depression and anxiety among adolescents during COVID-19: A crosssectional study. Brain Behav Immun 88:36-38. https://doi.org/10. 1016/j.bbi.2020.05.061

46. Petzold MB, Bendau A, Plag J, Pyrkosch L, Mascarell Maricic L, Betzler F et al (2020) Risk, resilience, psychological distress, and anxiety at the beginning of the COVID-19 pandemic in Germany. Brain Behav 10(9):e01745. https://doi.org/10.1002/brb3.1745

47. Spence SH, Sheffield J, Donovan C (2002) Problem-solving orientation and attributional style: moderators of the impact of negative life events on the development of depressive symptoms in adolescence? J Clin Child Adol Psychol 31(2):219-229. https://doi.org/ 10.1207/S15374424JCCP3102_07

48. Heiervang E, Goodman R (2011) Advantages and limitations of web-based surveys: evidence from a child mental health survey. Soc Psychiatry Psychiatr Epidemiol 46(1):69-76. https://doi.org/ 10.1007/s00127-009-0171-9

49. Hunersen K, Ramaiya A, Yu C, Green J, Pinandari AW, Blum $\mathrm{R}$ (2021) Considerations for remote data collection among adolescents during the COVID-19 pandemic. J Adolesc Health 68(3):439-440. https://doi.org/10.1016/j.jadohealth.2020.11.020
50. Goodman R (2013) Online child and adolescent mental health surveys can be good enough. Soc Psychiatry Psychiatr Epidemiol 48(8):1317-1325. https://doi.org/10.1007/s00127-013-0658-2

51. Morton S, Bandara D, Robinson E, Carr P (2012) In the 21st Century, what is an acceptable response rate? Aust N Z J Public Health 36(2):106-108. https://doi.org/10.1111/j.1753-6405.2012. 00854.x

52. Green KT, Beckham JC, Youssef N, Elbogen EB (2014) Alcohol misuse and psychological resilience among US Iraq and Afghanistan era veterans. Addict Behav 39(2):406-413. https://doi.org/10. 1016/j.addbeh.2013.08.024

53. Otto MW, Teachman BA, Cohen LS, Soares CN, Vitonis AF, Harlow BL (2007) Dysfunctional attitudes and episodes of major depression: Predictive validity and temporal stability in neverdepressed, depressed, and recovered women. J Abnorm Psychol 116(3):475-483. https://doi.org/10.1037/0021-843X.116.3.475

54. Rainey EE, Petrey LB, Reynolds M, Agtarap S, Warren AM (2014) Psychological factors predicting outcome after traumatic injury: the role of resilience. Am J Surg 208(4):517-523. https:// doi.org/10.1016/j.amjsurg.2014.05.016

55. Charles N, Strong SJ, Burns LC, Bullerjahn MR, Serafine KM (2021) Increased mood disorder symptoms, perceived stress, and alcoholic use among college students during the COVID-19 pandemic. Psychiatry Res 296:113706. https://doi.org/10.1016/j. psychres.2021.113706

56. Luthar SS, Ebbert AM, Kumar NL (2021) Risk and resilience during COVID-19: a new study in the Zigler paradigm of developmental science. Dev Psychopathol 33:565-580. https://doi.org/ 10.1017/S0954579420001388

57. Johnson D, Dupuis G, Piche J, Clayborne Z, Colman I (2018) Adult mental health outcomes of adolescent depression: a systematic review. Depress Anxiety 35(8):700-716. https://doi.org/ 10.1002/da.22777

58. Pine DS, Cohen P, Johnson JG, Brook JS (2002) Adolescent life events as predictors of adult depression. J Affect Disord 68(1):49 57. https://doi.org/10.1016/S0165-0327(00)00331-1

Publisher's Note Springer Nature remains neutral with regard to jurisdictional claims in published maps and institutional affiliations. 